\title{
ISOPERIMETRIC INEQUALITIES FOR POSITIVE SOLUTION OF P-LAPLACIAN
}

\author{
HUAXIANG HU AND QIUYi DAI
}

Abstract. In this paper, we prove some isoperimetric inequalities and give a explicit bound for the positive solution of P-Laplacian.

Mathematics subject classification (2010): 4QJ10, 35P15, 49J20.

Keywords and phrases: Isoperimetric inequality, Schwarz symmetrization, P-Laplacian, positive solution.

\section{REFERENCES}

[1] C. BANDLE, Isoperimetric inequality for some eigenvalues of an inhomogeneous free membrane, SIAM J. Appl. Math, 22, (1972), 2.

[2] M. S. Ashbaugh, R. D. Benguria, A sharp bound for the ratio of the first two eigenvalue of Dirichlet Laplacians and extensions, Ann. Math., 135, (1992), 601-628.

[3] QIUYi DaI, YUXIA FU, Faber-Krahn inequality for Robin problem involving p-Laplacian, Acta Mathematics Applicatae Sinica, English Series, 27, 1 (2011), 13-28.

[4] B. KAWOHL, V. FRIDMAN, Isoperimetric estimates for the first eigenvalue of the p-Laplace operator and the Cheeger constant, Comment. Math. Univ. Carolinae, 44, (2003), 659-667.

[5] T. BhatTACHARYA, A proof of the Faber-Krahn inequality for the first eigenvalue of the p-Laplacian, Annali di Matematica pura ed applicata, 177, IV (1999), 325-343.

[6] M. H. Bossel, Membranes élastiquement liées inhomogènes ou sur une surface: une nouvelle extension du théoréme isopérimétrique de Rayleigh-Faber-Krahn, Z. Angew. Math. Phys., 39, (1988), 733-742.

[7] D. Daners, A Faber-Krahn inequality for Robin problems in any space dimension, Math. Ann. 335, (2006), 767-785.

[8] G. FABER, Beweis, dass unter allen homogenen Membranen von gleicher Fläche und gleicher Spannung die kreisförmige den tiefsten Grundton gibt, Sitzungsber, Bayr. Akad. Wiss. München, Math.Phys. Kl, (1923), 169-127.

[9] E. KRAHN, Über eine von Rayleigh formulierte Minimaleigenschaft des Kreises, Math. Ann. 94, (1925), 97-100.

[10] E. KRAhn, Über Minimaleigenschaften der Kugel in drei und mehr Dimensionen, Acta Comm. Univ. Tartu (Dorpat), 9 (1926), 1-44.

[11] G. SZEGÖ, Inequalities for certain eigenvalues of a membrane of given area, J. Rational Mech. Anal., 3 (1954), 343-356.

[12] H. F. WEINBERGER, An isoperimetric inequality for the $n$-dimensional free membrane problem, J. Rational Mech. Anal., 5 (1956), 633-636.

[13] L. E. PAYNE, M. E. RAYNER, An isoperimetric inequality for the first eigenfunction in the fixed membrane problem, Journal of Applied Mathematics and Physics, 23, (1972), 13-15.

[14] L. E. PAYNE, M. E. RAYNER, Some isoperimetric norm bounds for solutions of the Helmholtz equation, Journal of Applied Mathematics and Physics, 24, (1973), 106-110.

[15] GiUseppe Chiti, A reverse Hölder inequality for the eigenfunctions of linear second order elliptic operators, Journal of Applied Mathematics and Physics, 33, (1982), 143-148.

[16] F. CHIACCHIO, Estimates for the first eigenfunction of linear eigenvalue problem via steiner symmetrization, Publ. Mat. 53, (2009), 47-71. 
[17] M. BeLloni, V. Ferone, B. KAWOHL, Isoperimetric inequalities, Wulff shape and related questions for strongly nonlinear elliptic operators, Z. angew. Math. Phys., 54 (2003), 771-783.

[18] M.-TH. KoHLER-JobIN, Isoperimetric monotonicity and Isoperimetric inequalities of Payne-Rayner type for the first eigenfunction of the Helmholtz problem, J. Appl. Math. Phys. (ZAMP), 32, (1981), 625-646.

[19] HuAXIANg Hu, QIUYi DAI, Renchu He, Isoperimetric Inequalities and Sharp Estimate for Positive Solution of Sublinear Elliptic Equations, Chinese Annals of Mathematics, 34A, 1 (2013), 87-100.

[20] M.-TH. KohleR-Jobin, Sur la premiè function propre d'une membrane: une extension à $N$ dimensions de l'inégalité isopérimétrique de Payne-Rayner, Z. angew. Math. Phys., 28, (1977), 1137-1140.

[21] H. F. Weinberger, Symmetrization in uniformly elliptic problems, Studies in Math. Anal., Stanford Uni. Press, 1962.

[22] G. TAlenti, Elliptic equations and rearrangements, Ann. Scuola Norm. Sup. Pisa, 3 (1976), $697-$ 718.

[23] C. Bandle, On symmetrization in parabolic equations, J. Anal. Math., 30, (1976), 98-112.

[24] G. TALENTI, Nonlinear elliptic equations, rearrangements of functions and orlicz spaces, Annali di Matematica Pura ed Applicata, 120, 1(1979), 0373-3114.

[25] B. Mess Ano, Symmetrization results for classes of nonlinear equations with q-growth in the gradient, Nonlinear Anlysis, 64, (2006), 2688-2703.

[26] A. Alvino, G. Trombetti And P.-L. Lions, Comparasion results for elliptic and parabolic equations via Schwarz symmetrization, Ann. Inst. Henri Poincaré, Analyse non linéaire, 7 (1990), 37-65.

[27] A. Alvino, P.-L. Lions And G. Trombetti, Comparasion results for elliptic and parabolic equations via Schwarz symmetrization: A new approach, Differ. Integral Equations, 4 (1992), 25-50.

[28] Robbert Osserman, The isoperimetric inequality, Bulletin of the American Mathematical Society, 84, 6 (1978).

[29] G. Pólya And G. SZEGÖ, Isoperimetric inequalities in mathematical physics, Ann. Math. Studies, 27, Princeton Univ. Press, 1951.

[30] L. E. PAYNE, Isoperimetric inequalities and their applications, SIAM Rev., 9 (1967), 453-483.

[31] L. E. PAYNE, Some comments on the past fifty years of isoperimetric inequalities, Lecture Notes in Pure and Appl. Math., 129, Dekker, New York, 1991.

[32] Tilak Bhattacharya, A Proof of the Faber-Krahn Inequality for the First Eigenvalue of the pLaplacian, Annali di Matematica pura ed applicata (IV), CLXXVII, (1999), 225-240.

[33] S. Kesavan, F. Pacella, Symmetry of positive solutions of a quasilinear elliptic equation via isoperimetric inequalities, Applicable Anal., 54, (1994), 27-37.

[34] J. Mossino, A generalization of the Payne - Rayner isoperimetric inequality, Boll. Un. Mat. Ital., 23, 6 (1983), 335-342.

[35] G. H. Hardy, J. E. Littlewood and G. Pólya, Some simple inequalities satisfied by convex functions, Messenger Math., 58, (1929), 152.

[36] Hu HuAXIANG, ZHOU SHUQIng, Brunn-Minkoski inequality for variational functional involving the P-Laplacian operator, Acta Math., 29 B, 5 (2009), 1143-1154. 